\title{
Benign expectoration of a surgical clip through a pneumonectomy stump
}

\author{
Zahoor Ahmed, MD, Larry R. Kaiser, MD, and Joseph B. Shrager, MD, Philadelphia, Pa
}

A lthough technologic advances have brought considerable convenience and timesaving to a number of thoracic surgical procedures, such advances may also be the source of unusual complications. Bronchial arteries, which formerly would have been ligated, are now often controlled by electrocautery or the application of surgical clips. We report what we believe to be the previously unreported complication of expectoration of a titanium surgical clip through a right pneumonectomy stump, pneumonectomy having been performed several years previously for a destroyed, bronchiectatic lung.

\section{Clinical Summary}

A 63-year-old woman had undergone right upper lobectomy at an overseas institution 32 years previously. Although detailed information about this procedure is unavailable, the patient reported that the procedure was carried out for resection of a "lymphoma." She received postoperative radiotherapy and was apparently cured of this disorder. During the subsequent 32 years, cystic bronchiectatic changes that gradually progressed to complete destruction of the pulmonary parenchyma developed in the remaining right lung, with cough and sepsis resistant to medical therapy. We performed a completion pneumonectomy in 1997, and the bronchial closure was carried out with a mechanical stapler and reinforced with transposed serratus anterior and latissimus dorsi muscle flaps, which served also to fill the pneumonectomy space. As a result of the chronic inflammatory process in the chest, there were numerous dilated bronchial arteries present, and these were controlled with surgical clips. The patient's postoperative course was uneventful, and she was discharged home on the fifth postoperative day.

Six years later, the patient had a sudden bout of coughing, and she expectorated a surgical clip (Figure 1). She also acquired vague discomfort over the right scapular region at this time, but she had no fever, hemoptysis, dyspnea, or dysphagia. When we saw the patient 3 weeks later, there were no signs or symptoms suggestive of a pleural space infection. Laboratory data, including

From the Section of General Thoracic Surgery, Division of Cardiothoracic Surgery, University of Pennsylvania School of Medicine, Philadelphia, Pa.

Received for publication Feb 19, 2002; accepted for publication March 2, 2002.

Address for reprints: Joseph B. Shrager, MD, Chief, General Thoracic Surgery, University of Pennsylvania School of Medicine, 4 Silverstein Pavilion, 3400 Spruce St, Philadelphia, PA 19104-4283 (E-mail: j.shrag@mail.med.upenn.edu).

J Thorac Cardiovasc Surg 2002;124:1025-6

Copyright (C) 2002 by The American Association for Thoracic Surgery $0022-5223 / 2002 \$ 35.00+0 \quad \mathbf{1 2 / 5 4 / 1 2 4 4 9 5}$

doi: $10.1067 / \mathrm{mtc} .2002 .124495$ white blood cell count, were within normal limits. Chest radiography, however, demonstrated a right apical air space that we believed might signify either a bronchopleural fistula or simple herniation of the left lung across the midline (Figure 2). No previous films, other than her immediate postoperative films, were available for comparison because she lived overseas. We ordered a computed tomographic scan of the chest and a subsequent barium swallow (Figure 3), which revealed that the right apical air space represented a markedly dilated proximal esophagus without distal obstruction.

Bronchoscopy demonstrated a healed pneumonectomy stump without evidence of an actual, open bronchopleural fistula but with another clip apparently working its way into the airway, with approximately half of the clip visible within the lumen. The side of the clip that would be open before closure by the surgeon formed the leading edge of the clip visible in the lumen. We opted not to manipulate the clip bronchoscopically. The patient was told that it was likely that she would cough out another clip at some point in the future, but that this was nothing about which she should be concerned as long as she did not have fevers or otherwise become ill.

\section{Discussion}

There is little information in the surgical literature regarding the expectoration of foreign materials after pulmonary surgery. Two of the previous reports concerned lung volume reduction surgery, ${ }^{1,2}$ and the expectorated materials in both consisted of staples and dry bovine pericardial strips used for staple line reinforcement. Inflammation initiated by the staples or more likely the buttressing material is speculated to have resulted in erosion of small bronchioles and the subsequent expectoration. Saunders and coworkers $^{3}$ reported a case of bullet migration through pulmonary parenchyma and its spontaneous expulsion. We are not, however, aware of any previous report of nonreactive, commonly used titanium surgical clips eroding into the bronchial tree.

Bronchiectasis, by virtue of the severe inflammatory process that it creates, is accompanied by dilated bronchial arteries and dense, vascular adhesions. These changes render surgical dissection tedious and increase the propensity toward bleeding. Ligation of all bronchial arteries in these circumstances with ties is timeconsuming, and many of the vessels are too large to control with cautery. Particularly in the vicinity of the bronchial stump, the use of cautery, with its risk of spreading heat injury, is ill-advised. Control of bleeders with titanium clips in these areas is technically easy and has been considered a relatively safe option. Many surgeons use these clips routinely in the control of bronchial arteries.

In the case presented here, the constant motion of the bronchial stump during respiratory cycle through the years appears to have resulted in the slow migration of these fairly sharp surgical clips from the small bronchial vessels in the vicinity of the right main 


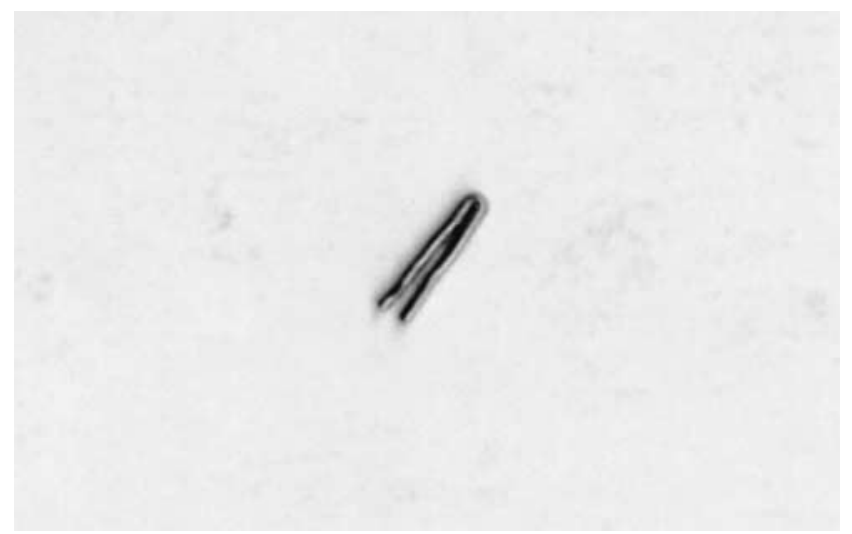

Figure 1. Photograph of actual titanium clip expectorated by patient.

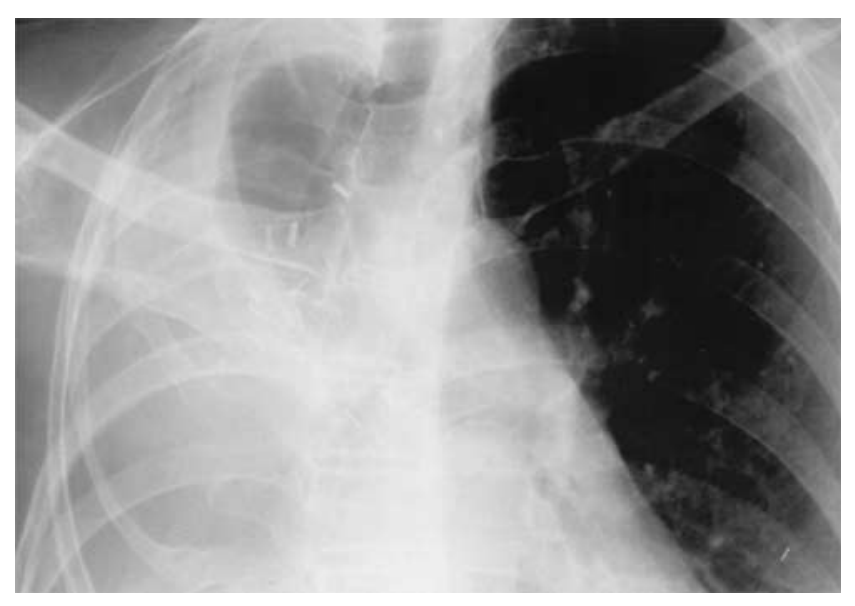

Figure 2. Chest radiograph demonstrating air space adjacent to the right pneumonectomy stump. Note multiple radiopaque clips also in the vicinity of the stump.

bronchial stump into the airway. An ongoing inflammatory process does not appear to have contributed, because no signs indicative of inflammation were discovered locally in the right bronchial stump during bronchoscopy, nor was there any systemic evidence of infection.

The appearance of the chest radiograph was deceptive and increased our concern that an actual bronchopleural fistula had occurred. Chest computed tomography suggested the true nature of the suspicious air in the right pleural cavity, and this was confirmed by barium swallow to represent dilated esophagus. The esophageal dilation had likely been caused by traction on the esophageal wall related to fibrosis within the thoracic cavity, because no distal obstruction was identified.

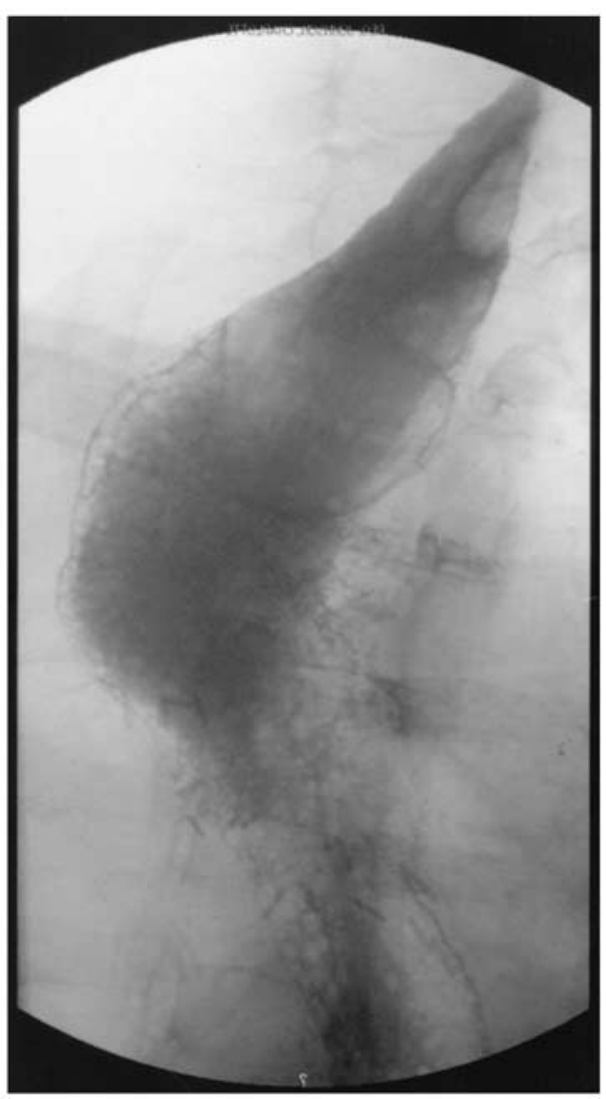

Figure 3. Barium swallow confirming dilated proximal esophagus without distal obstruction.

This is the first report of expectoration of a titanium surgical clip after a thoracic surgical procedure. The clip appears to have gradually eroded through a pneumonectomy stump after being used to control bronchial arteries in the vicinity of the stump. There was no associated bronchopleural fistula permitting passage of air or microbes. The occurrence of this event highlights that surgical clips should be used with great care in the vicinity of the bronchial stump and further suggests that perhaps they should be redesigned such that their ends are blunt rather than sharp.

\section{References}

1. Oey I, Walker DA. Metalloptysis: a late complication of lung volume reduction surgery. Ann Thorac Surg. 2001;71:1695-7.

2. Ahmed S, Marzouk KA, Bhuiya TA, Iqbal M, Rossoff LJ. Asymptomatic expectoration of surgical staples complicating lung volume reduction surgery. Chest. 2001;119:307-8.

3. Saunders MS, Cropp AJ, Awad MJ. Spontaneous endobronchial erosion and expectoration of a retained intrathoracic bullet Trauma. 1992;33:909-11. 